\title{
Vielfachstreuung schneller Elektronen
}

\author{
Von O. KNeCHT* und W. Bothe \\ Aus dem Institut für Physik im Max-Planck-Institut für med. Forschung, Heidelberg \\ (Z. Naturforschg. 8a, 805-814 [1953]; eingegangen am 3. Oktober 1953)
}

\begin{abstract}
Aus dem $\beta$-Spektrum des RaE wurden homogene Parallelbündel in dem Energiebereich $250-1000 \mathrm{keV}$ ausgesondert und deren Vielfachstreuung in Metallfolien (Al, Ni, $\mathrm{Ag}, \mathrm{Au}$ ) von verschiedener Dicke gemessen. Die Ergebnisse über die Form und Breite der Winkelverteilung hinter der Folie wurden mit den verschiedenen Theorien der Vielfachstreuung verglichen. Es ergab sich vollständige Übereinstimmung mit der Theorie von Molière in deren Gültigkeitsbereich. Für die praktische Ermittlung der Verteilungsbreite, auch außerhalb der Gültigkeitsgrenzen der bisherigen Theorien, wird ein einfaches Verfahren angegeben. Weitere Messungen wurden über die Winkelverteilung hinter schräg gestellten Folien gemacht. Die Ergebnisse bestätigen die Berechnungen innerhalb deren Gültigkeitsbereich.
\end{abstract}

\section{Zielder Untersuchung \\ und theoretische Formeln}

$\mathrm{D}_{\mathrm{s}}^{\mathrm{ic}}$ Gesetze der Coulomb-Vielfachstreuung schneller geladener Teilchen lassen sich grundsätzlich durch rein mathematische Operationen aus den Gesetzen der Einzelstreuung herleiten. Es zeigt sich jedoch, daß diese Rechnungen um so verwickelter und die Ergebnisse um so unhandlicher werden, je höher die Ansprüche an Genauigkeit sind. Daher sind mehrere Näherungstheorien aufgestellt worden $^{1-7}$. Genaue Messungen der Vielfachstreuung sind jedoch verhältnismäßig spärlich ${ }^{8,9}$. Es schien daher angebracht, das Problem der Vielfachstreuung von Elektronen erneut von der experimentellen Seite anzugreifen, erstens um die bestehenden Theorien in größerem Umfange nachzuprüfen, zweitens um experimentelle Unterlagen für eine einfache Vorausbestimmung der Vielfachstreuung bei mäßigen Genauigkeitsansprüchen zu gewinnen.

Alle Theorien der Vielfachstreuung ergeben in Übereinstimmung mit der Erfahrung, daß bis zu einem gewissen Streuwinkel die räumliche Winkelverteilung der gestreuten Teilchen durch eine GaußFunktion gut dargestellt werden kann:

\footnotetext{
* Diss. Heidelberg 1953.

${ }^{1}$ W. Bothe, Z. Physik 4, 300 [1931]; 5, 63 [1921]; Handb. d. Physik XXIV, 6 [1927].

2 E. J. Williams Proc. Roy. Soc. [London] Ser. A 169, 531 [1939]; Physic. Rev. 5̌8, 292 [1940].

3 S. Goudsmit u. J. L. Saund erson, Physic. Rev. 57, 24 [1940]; 58, 36 [1940].

4 G. Molière, Z. Naturforschg. 2a, 133 [1947]; 3a, 78 [1948].

5 H. S. Snyder u. W. T. Scott, Physic. Rev. 76, 220 [1949].
}

$$
p(\Theta) \mathrm{d} \Omega=\frac{d \Omega}{2 \pi \lambda^{2}} \exp \left(-\frac{\Theta^{2}}{2 \lambda^{2}}\right) .
$$

Hierin ist $\Theta$ der tatsächliche, $\lambda$ der wahrscheinlichste Streuwinkel, d $\Omega$ das Raumwinkelelement. Bei großen Winkeln wird die Streuintensität größer als nach Gl. (1), weil sich Mehrfach- und Einzelstreuung überlagern. Indessen erweist sich die Gaußsche Näherung in vielen Fällen als ausreichend. Sie wird durch einen einzigen Parameter $\lambda$ festgelegt, oder auch durch den ,Halbwertwinkel“ $\Theta_{\mathrm{H}}=1,18 \lambda$, bei dem die Streuintensität gerade halb so groß ist wie im Durchstoßpunkt. Eine einfache Näherungsformel für $\lambda$ wurde schon 1921 unter sehr vereinfachenden Annahmen über die Abschirmung der streuenden Atomkerne durch die Elektronenhülle hergeleitet $^{1}$

$$
\lambda=\frac{C}{E} \frac{E+E_{0}}{E+2 E_{0}} Z \sqrt{\frac{\varrho x}{A}},
$$

wo $E$ die kinetische, $E_{0}$ die Ruheenergie des Teilchens ist, $Z$ die Ordnungszahl, $A$ das Atomgewicht, $\varrho$ die Dichte $\left(\mathrm{g} / \mathrm{cm}^{3}\right), x$ die Schichtdicke $(\mathrm{cm})$ der streuenden Schicht. $C$ ergab sich in dieser Näherung für alle einfach geladenen Teilchen als eine Konstante $(C \approx 0,8 \mathrm{MeV}$ nach den wenigen damals vor-

\footnotetext{
${ }^{6}$ W. T. Sc ott, Physic. Rev. 85, 245 [1952].

7 Die Theorie von Snyder u. Scott ${ }^{5}$ hat sich im wesentlichen als identisch mit derjenigen von Moli èr ${ }^{4}$ erwiesen $^{6}$ und braucht daher im folgenden nicht weiter herangezogen zu werden.

${ }^{8}$ L. Kulchitsky u. G. Latyshev, Physic. Rev. 61, 254 [1942].

9 A. O. Hanson, L. H. Lanzl, E. M. Lyman u. M. B. Scott, Physic. Rev. \$4, 634 [1951].
} 
liegenden Messungen), jedoch zeigten die späteren genaueren Theorien und Experimente, daß $C$ eine langsam veränderliche Funktion von $E, Z$ und $\varrho x$ ist. Diese Funktion experimentell zu bestimmen, bildete das zweite der oben angedeuteten Ziele der Untersuchung.

Im Zusammenhang mit diesen Messungen wurde schließlich noch ein Problem bearbeitet, das bisher theoretisch nur in grober Näherung, experimentell überhaupt nicht behandelt worden ist, nämlich die Vielfachstreuung von Elektronen beim schrägen Durchgang durch Folien. Die theoretische Gaußsche Näherung lautet für diesen Fall ${ }^{10}$ :

$$
\begin{aligned}
p(\varphi, \psi) \mathrm{d} \Omega & =\frac{d \Omega}{2 \pi \lambda^{2}} \exp \left(-\frac{\varphi^{2}+\psi^{2}}{2 \lambda^{2}}\right) \\
& \cdot\left[1-\psi \operatorname{tg} \alpha\left(1-\frac{\varphi^{2}+\psi^{2}}{4 \lambda^{2}}\right)\right] .
\end{aligned}
$$

Hierin ist $\alpha$ der Neigungswinkel der Folie, $\varphi$ und $\psi$ sind die (kleinen) rechtwinkligen Koordinaten des Streuwinkels, wobei $\psi$ parallel zur Einfallsebene gerichtet ist; $\lambda$ ist der wahrscheinlichste Streuwinkel, wie er bei senkrechtem Durchgang durch eine Schicht von derselben effektiven Dicke (Dicke/cos $\alpha$ ) auftreten würde. legt. Die Elektronen konnten durch ein Glimmerfenster von $4,8 \mathrm{mg} / \mathrm{cm}^{2}$ in die Monochromatorkammer eintreten. Die ausgesonderten Elektronenenergien lagen zwischen 0,25 und $1 \mathrm{MeV}$. Die benötigten magnetischen Feldstärken lagen alle im Bereich des linear ansteigenden Teils der Hysteresis. Durch Shimringe S am Rande der Polflächen wurde der Feldabfall steiler gemacht. Vor jeder Messung wurde die magnetische Remanenz durch Stromwenden beseitigt. Der Druck in den Kammern betrug bei den Versuchen höchstens $10^{-3}$ Torr. Das Blendenrohr I bestand aus Eisen. Die drei kreisrunden Blenden aus Blei, $B_{1}, B_{2}, B_{3}$ hatten einen lichten Durchmesser von $0,4 \mathrm{~cm}$. Sie wurden zeitweise durch größere Blenden von $1,0 \mathrm{~cm}$ Durchmesser ersetzt, wenn eine größere einfallende Elektronenintensität notwendig war. Die Blendenränder waren abgeschrägt.

Die Streukammer K bestand in der Hauptsache aus einem vakuumdichten Tombakschlauch TS, der an das Blendenrohr I montiert war. In der Streukammer befand sich die Streufolie F. Sie war auf einen runden Messingrahmen von 2,7 cm Durchmesser aufgespannt, der mit Hilfe zweier Stellschrauben in dem Haltestück FH befestigt war. Das Haltestück war in das Blendenrohr I um dessen Achse drehbar eingesetzt. Das drehbare Haltestück und die beiden Stellschrauben bildeten ein Cardangehänge, so daß die Folie beliebig um ihren Mittelpunkt geschwenkt werden konnte. Der ganze Teil rechts von der Folie, also das Blendenrohr II mit dem Zählrohr ZR, konnte um die zwei Drehbolzen

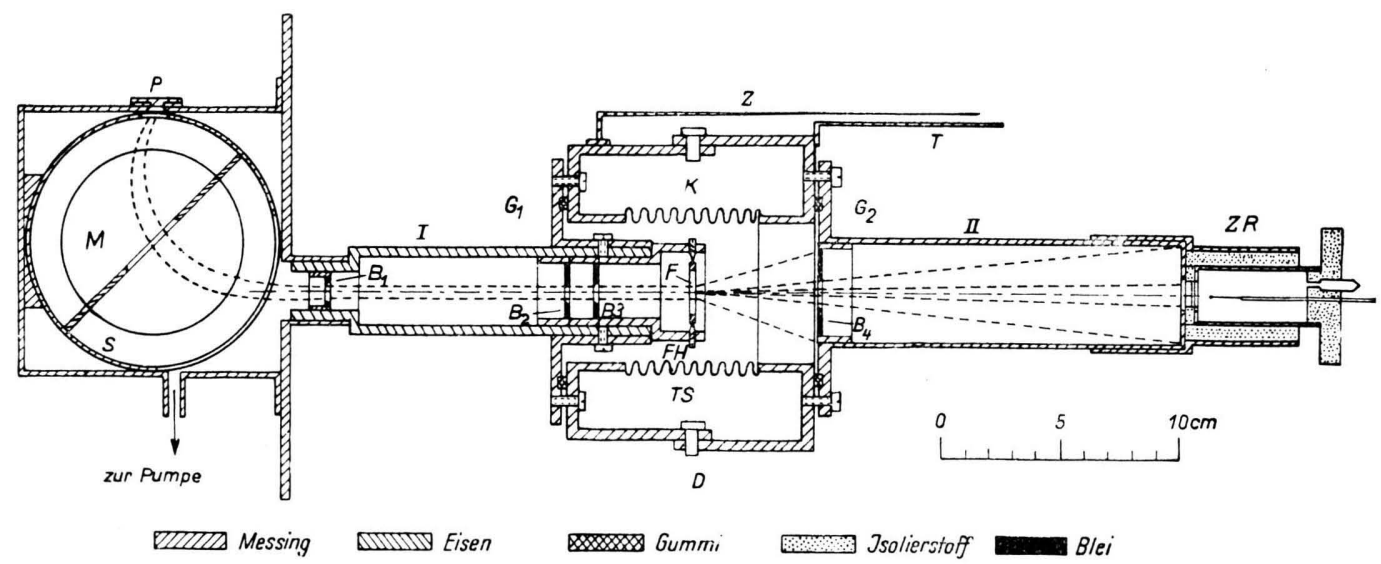

Abb. 1. Längsschnitt durch die Versuchsanordnung.

\section{Versuchsanordnung (A bb. 1)}

Die Apparatur bestand aus dem Monochromator M mit Blendenrohr I, der Streukammer K mit dem Blendenrohr II und der Zähleinrichtung.

Der kolliminierende Monochromator ist schon an früherer Stelle ${ }^{11}$ ausführlich beschrieben worden. Ein etwa $30 \mathrm{mC}$ starkes $\mathrm{RaD}+$ E-Präparat $P$ war senkrecht über der Polachse des Elektromagneten aufge-

${ }^{10} \mathrm{~W}$. Bothe, S.-B. Heidelb. Akad. 1951, 7.Abh., 307.
D geschwenkt werden. Es wurde darauf geachtet, da $\beta$ die Drehachse durch die Folienmitte lief, so daß der Abstand Folie-Zählrohr bei einer Drehung konstant blieb. Der Tombakschlauch erlaubte eine Auslenkung des drehbaren Teils $u m \pm 50^{\circ}$. Der Zeiger $\mathrm{Z}$ war am festen Teil montiert, der Skalentisch $\mathrm{T}$ am drehbaren Teil. Bei Folienwechsel wurde das Blendenrohr II an der Gummidichtung $G_{2}$ abgeschraubt. Im Blendenrohr II war die Bleiblende $B_{4}$ (Durchmesser $1,0 \mathrm{~cm}$ )

${ }^{11}$ W. Bothe, Z. Naturforschg. 4 a, 88 [1949]. 
nächst der Streufolie eingesetzt, um Streuung an der Wand des Blendenrohres II zu verhindern. Aus demselben Grund war die ganze Apparatur noch mit $\mathrm{Pa}$ raffinpapier ausgekleidet. Das Ende des Blendenrohres II hatte ein kreisrundes Austrittsfenster (Glimmer 2,5 mg/ $\mathrm{cm}^{2}$, Durchmesser 0,8 cm). Das Zählrohr ZR konnte in ein isolierendes Haltestück so eingeschoben werden, daß sich Zählerfenster und Austrittsfenster dicht gegenüberlagen.

Die Gold- und Silberfolien mit einer Dicke $\geqq 5 \cdot 10^{-4}$ cm, ebenfalls alle Aluminiumfolien waren Handelsware. Die dünneren Gold- und Silberfolien (bis herab $\mathrm{zu} 6 \cdot 10^{-5} \mathrm{~cm}$ ) und die Nickelfolien wurden nach altbewährten Badrezepten ${ }^{12}$ galvanisch auf Kupfer niedergeschlagen. Zum Ablösen der Kupferfolien von den Gold- und Nickelfolien wurde konzentrierte Chromsäure benutzt, die mit etwas Schwefelsäure aktiviert worden war. Dieses Verfahren konnte bei der Herstellung von Silberfolien nicht angewandt werden, da auch Silber von diesem Lösungsmittel angegriffen wird. Man mußte sich mit einer Kupfertetraminsulfatlösung ( 1 Teil konz. $\mathrm{Cu}\left(\mathrm{NH}_{3}\right)_{4} \mathrm{SO}_{4}+2$ Teile $\mathrm{H}_{2} \mathrm{O}$ ) begnügen, die nur verhältnismäßig langsam das Kupfer vom Silber ablöste. Die Dickenbestimmung der Folien geschah durch Wägen und Planimetrieren auf $1 \%$ genau. Die Folien waren genügend rein.

Die Zähleinrichtung bestand aus Spitzenzähler ZR, Hochspannungsgerät mit Röhrenstabilisator und Impulsverstärker mit Zählwerk. Der Spitzenzähler hatte 2,5 cm Durchmesser. Die Spitze war ein Platinkügelchen von $0,1 \mathrm{~mm}$ Durchmesser an einem 0,05 mm Platindraht. Die Entfernung der Spitze vom Zählrohrfenster betrug $0,8 \mathrm{~cm}$. Das Füllgas bestand aus 70 Torr Argon und 20 Torr Alkoholdampf. Die Einsatzspannung betrug 850 Volt, die Länge des Plateaus der Charakteristik etwa 100 Volt. Das Zählrohrfenster hatte $1,0 \mathrm{~cm}$ Durchmesser und bestand aus $2 \mathrm{mg} / \mathrm{cm}^{2}$ Glimmer. Außerdem wurde zeitweise ein Stirnfensterzählrohr benutzt, das durch ein besseres Plateau und ein größeres Zählvolumen ausgezeichnet war, aber etwas höheren Nulleffekt hatte. Hochspannungsgerät und Spannungsstabilisator waren nach altbekanntem Muster hergestellt. Der Impulsverstärker hatte drei Stufen: Multivibrator, eine Untersetzerstufe (2-fach) und Endstufe. Die Auflösekorrektur betrug $2 \%$ bei $1000 \mathrm{Stößen} / \mathrm{min}$. Der Nulleffekt konnte durch Bleiabschirmung des $\mathrm{RaD}+$ E-Präparates auf 12/min (Spitzenzähler) bzw. 25/min (Stirnfensterzählrohr) herabgedrückt werden. Bei den Hauptmessungen wurden immer 8000-10000 Elektronen pro Meßpunkt gezählt, so daß der mittlere statistische Fehler etwa $1 \%$ betrug. Es wurden nur selten mehr als 1000 Elektronen/min gezählt.

\section{Vorversuche}

Der Monochromator wurde mit dem $\beta$-Spektrum eines RaEm-Hütchens geeicht, das mit Glimmer von $1 \mathrm{mg} / \mathrm{cm}^{2}$ verschlossen war. Als Eichpunkt wurde die scharfe Linie $1938 \Gamma \mathrm{cm}$ von $\mathrm{RaB}$ gewählt. Die

12 Galvanotechnik (Früher Pfanhauser), Leipzig 1949 Bd. I, 453, 908 .
Halbwertbreite der Linien betrug etwa 4\%. Die Glimmerfenster des Hütchens und des Monochromators trugen dabei wesentlich zur Verbreiterung der Linien bei. Man kann annehmen, daß die Energiehalbwertbreite des Elektronenstrahls, der bei den späteren Messungen aus dem kontinuierlichen RaESpektrum stammte, dann entsprechend der endlichen Öffnung der Blenden etwa $2-3 \%$ betrug. Mit Hilfe der wohldefinierten Grenze des kontinuierlichen RaE-Spektrums wurde die Eichung kontrolliert. Die Eichungen stimmten auf etwa $1 \%$ überein.

Als weitere Frage von Wichtigkeit erwies sich die Intensitätsverteilung im primären Elektronenstrahl. Es wurde die Versuchsanordnung nach Abb. 1 ohne Folie benutzt. Vor dem Zählerfenster war eine Messingblende von $0,1 \mathrm{~cm}$ Durchmesser angebracht. Durch Variation der Winkelstellung des Zählrohres konnte also der Primärstrahl abgetastet werden.

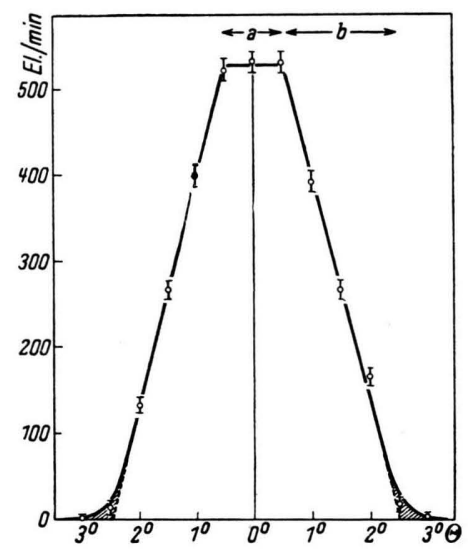

Abb. 2. Intensitätsverteilung im Primärstrahl bei $0,4 \mathrm{~cm}$-Blenden $\mathbf{B}_{1}, \mathbf{B}_{2}, \mathbf{B}_{3}$.

Dies wurde für drei Strahlenbündeldurchmesser $(0,2 ; 0,4 ; 1,0 \mathrm{~cm})$ durchgeführt. Für den Durchmesser $0,4 \mathrm{~cm}$ ist die Intensitätsverteilung in Abb. 2 dargestellt. Die Fläche der kleinen schraffierten Schwänze in Abb. 2 ist ein Maß für die an den Rändern von $\mathrm{B}_{2}$ und $\mathrm{B}_{3}$ gestreuten Elektronen. Sie ist weniger als $1 \%$ der Gesamtfläche der Kurve. Für verschiedene Elektronenenergien blieben diese kleinen Flächen gleich groß. Sie konnten bei den späteren Streumessungen vernachlässigt werden.

Nicht vernachlässigbar war die endliche Breite des Primärstrahls; sie vergrößerte allgemein den mittleren Streuwinkel hinter den Folien bei den späteren Messungen. Die Halbwertbreite der Kurve 
in Abb. 2 ist hauptsächlich durch die „Halbschatten" $b$ bedingt. Die kurze Trapezseite $a$ entspricht dem Blendendurchmesser von $0,4 \mathrm{~cm}$. Während die Seite $a$ proportional mit dem Durchmesser der Blenden $B_{1}, B_{2}$ und $B_{3}$ ging, blieb oberhalb $0,4 \mathrm{~cm}$ der „Halbschatten“ $b$ praktisch gleich breit. Hierin kommt die kollimierende Eigenschaft des Monochromators zum Ausdruck. Wurde die enge Blende vor dem Zählrohr weggenommen und damit der vom Zählrohr erfaßte Raumwinkel vergrößert, so entstand aus der Kurve Abb. 2 eine Glockenkurve mit praktisch derselben Halbwertbreite und mittleren Breite.

Die Streuverteilung hinter einer Folie mußte hinsichtlich ihres Halbwertwinkels infolge der endlichen Primärstrahldicke folgendermaßen korrigiert werden:

$$
\Theta_{\mathrm{H}}^{2}=\Theta_{\mathrm{H}}^{* 2}-\Theta_{\mathrm{H}}^{02}
$$

Gl. (4) folgt, wenn man nicht nur die Streuverteilung, sondern auch die Nullverteilung Abb. 2 durch eine Gauß-Verteilung mit entsprechender mittlerer Ablenkung annähert. In Gl. (4) bedeuten: $\Theta_{\mathrm{H}}^{*}=$ gemessener Halbwertwinkel, $\Theta_{\mathrm{H}}=$ korrigierter Halbwertwinkel, $\Theta_{\mathrm{H}}^{0}=$ Halbwertwinkel der angenäherten Gauß-Verteilung im Primärstrahl. Es gilt $\Theta_{\mathrm{H}}^{0}=0,84 \sqrt{{\overline{\Theta^{02}}}^{2}}$, wenn $\overline{\Theta^{02}}$ aus Abb. 2 gewonnen wird. Es war $\Theta_{\mathrm{H}}^{0}=0,9^{0}$ bei einem Blendendurchmesser von $0,2 \mathrm{~cm}$ von $\mathrm{B}_{1}, \mathrm{~B}_{2}, \mathrm{~B}_{3} ; \Theta_{\mathrm{H}}^{0}=1,5^{0}$ bei $0,4 \mathrm{~cm}$ Blendendurchmesser; $\Theta_{\mathrm{H}}^{0}=2,2^{0}$ bei $1,0 \mathrm{~cm}$ Blendendurchmesser. Die Korrektionen nach (4) betrugen bei allen späteren Messungen weniger als $2 \%$. Gl. (4) wurde durch Variation der Dicke des Primärstrahls bei eingesetzter Folie mit einem $\Theta_{\mathrm{H}}=5,06$ bestätigt.

\section{Vielfachstreuung als Funktion der Foliendicke}

Die in diesem Abschnitt behandelten Messungen wurden durchweg mit $860 \mathrm{keV}$-Elektronen durchgeführt. Die Dicke $x$ von Al-, Ni-, Ag- und Au-
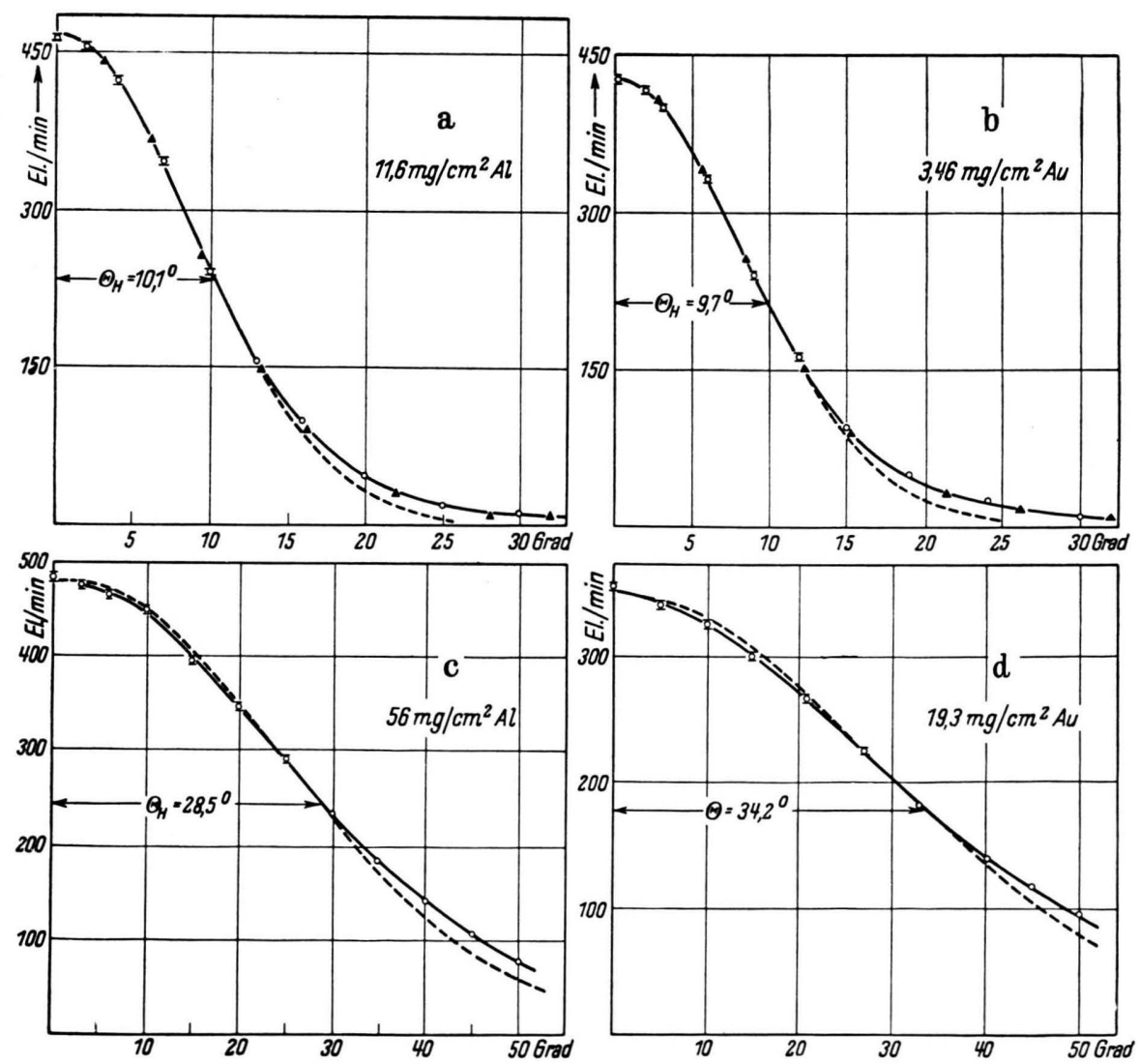

Abb. 3. Einige Beispiele der Winkelverteilungskurven für $860 \mathrm{keV}$-Elektronen. Als Abszisse wurde der Streuwinkel in Grad, als Ordinate die Elektronenzahl/min aufgetragen. - $\overline{\overline{ }}$ experimentell, - - . - Gauß-Kurve, $\Delta$ Molière. 
Folien wurde so variiert, daß Winkelverteilungskurven mit Halbwertwinkeln zwischen $5^{0}$ und $45^{\circ}$ herauskamen. Einige Beispiele der Verteilungskurven sind in Abb. 3 dargestellt. Die Korrektionen wegen der Nullbreite (Ziff. 3) sind hier fast zu vernachlässigen, sie wurden jedoch beim Zeichnen der Kurven berücksichtigt. Man sieht, daß in den Fällen a und $b$, wo die Streuung verhältnismäßig schwach ist, die experimentellen Verteilungskurven vollkommen durch die Theorie von Molière wiedergegeben werden. Dasselbe zeigte sich in allen Fällen, wo die Voraussetzungen dieser Theorie erfüllt waren. Dagegen traten in den Fällen $\mathrm{c}$ und d der Abb. 3, wo mit den theoretischen ist zu beachten, da $\beta$ aus verschiedenen Gründen die bestehenden Theorien nicht unmittelbar auf Verteilungen mit großem Halbwertwinkel angewandt werden können. Einer dieser Gründe, auf den Goudsmit und Saunderson ${ }^{3}$ schon hingewiesen haben, ist der, daß die für die Verteilungsbreite maßgebende Bahnlänge $l$ des Teilchens in der Schicht größer ist als die Schichtdicke $x$. Man kann $l$ auf einfache Weise überschlagsmäßig berechnen. Es sei $\vartheta$ der Winkel, um den das Bahnelement $\mathrm{d} l$ in der Tiefe $\xi$ gegen die Schichtnormale geneigt ist. Dann ist, Gauß-Verteilung vorausgesetzt,*

\begin{tabular}{|c|c|c|c|c|c|c|c|c|c|}
\hline \multicolumn{5}{|c|}{ Al } & \multicolumn{5}{|c|}{$\mathrm{Ni}$} \\
\hline 1 & 2 & 3 & 4 & 5 & 6 & 7 & 8 & 9 & 10 \\
\hline$\varrho x \cdot 10^{3}$ & $\Theta_{\mathrm{H}}$ & $\Theta_{\mathrm{H}}^{\mathrm{korr}}$ & $\Theta_{\mathrm{H}}^{\mathrm{korr}} / \Theta_{\mathrm{H}}^{\mathrm{M}}$ & $\Theta_{\mathrm{H}}^{\mathrm{korr}} / \Theta_{\mathrm{H}}^{\mathrm{GS}}$ & $\varrho x \cdot 10^{3}$ & $\Theta_{\mathrm{H}}$ & $\Theta_{\mathrm{H}}^{\mathrm{krrr}}$ & $\Theta_{\mathrm{H}}^{\mathrm{korr}} / \Theta_{\mathrm{H}}^{\mathrm{M}}$ & $\Theta_{\mathrm{H}}^{\mathrm{korr}} / \Theta_{\mathrm{H}}^{\mathrm{GS}}$ \\
\hline $\begin{array}{c}2,27 \dagger \\
4,72 \\
11,6 \\
23,2 \\
38,4 \dagger \\
56,0 \dagger \\
89,6 \dagger \\
146 \dagger\end{array}$ & $\begin{array}{c}3,45 \\
5,60 \\
10,1 \\
15,3 \\
22,3 \\
28,5 \\
38,5 \\
48,0\end{array}$ & $\begin{array}{c}3,45 \\
5,60 \\
10,0 \\
15,1 \\
21,7 \\
27,3 \\
35,4 \\
42,2\end{array}$ & $\begin{array}{l}1,14 \pm 0,04 \\
1,00 \pm 0,02 \\
1,00 \pm 0,02 \\
1,00 \pm 0,02 \\
1,06 \pm 0,02 \\
1,08 \pm 0,02 \\
1,07 \pm 0,03 \\
0,98 \pm 0,04\end{array}$ & $\begin{array}{l}0,97 \pm 0,04 \\
1,00 \pm 0,02 \\
1,02 \pm 0,02 \\
1,03 \pm 0,02 \\
1,10 \pm 0,02 \\
1,12 \pm 0,02 \\
1,10 \pm 0,03 \\
1,02 \pm 0,04\end{array}$ & $\begin{array}{l}1,93 \\
10,3 \\
30,9 \dagger\end{array}$ & $\begin{array}{l}4,60 \\
14,1 \\
29,5\end{array}$ & $\begin{array}{l}4,60 \\
13,9 \\
28,0\end{array}$ & $\begin{array}{l}1,00 \pm 0,03 \\
1,00 \pm 0,02 \\
1,07 \pm 0,02\end{array}$ & $\begin{array}{l}1,01 \pm 0,03 \\
1,00 \pm 0,02 \\
1,08 \pm 0,02\end{array}$ \\
\hline \multicolumn{5}{|c|}{$\mathrm{Ag}$} & \multicolumn{5}{|c|}{$\mathrm{Au}$} \\
\hline 11 & 12 & 13 & 14 & 15 & 16 & 17 & 18 & 19 & 20 \\
\hline$\varrho x \cdot 10^{3}$ & $\Theta_{\mathrm{H}}$ & $\Theta_{\mathrm{H}}^{\text {korr }}$ & $\Theta_{\mathrm{H}}^{\mathrm{korr}} / \Theta_{\mathrm{H}}^{\mathrm{M}}$ & $\Theta_{\mathrm{H}}^{\mathrm{korr}} / \Theta_{\mathrm{H}}^{\mathrm{GS}}$ & $\varrho x \cdot 10^{3}$ & $\Theta_{\mathrm{H}}$ & $\Theta_{\mathrm{H}}^{\mathrm{korr}}$ & $\Theta_{\mathrm{H}}^{\mathrm{korr}} / \Theta_{\mathrm{H}}^{\mathrm{Y}}$ & $\Theta_{\mathrm{H}}^{\mathrm{knar}} / \Theta_{\mathrm{H}}^{\mathrm{GS}}$ \\
\hline $\begin{array}{r}1,52 \\
3,65 \\
5,73 \\
11,1 \dagger \\
17,2 \dagger \\
32,0 \dagger\end{array}$ & $\begin{array}{l}4,85 \\
8,70 \\
11,7 \\
19,0 \\
24,8 \\
38,2\end{array}$ & $\begin{array}{l}4,85 \\
8,65 \\
11,6 \\
18,6 \\
24,0 \\
35,1\end{array}$ & $\begin{array}{l}1,03 \pm 0,03 \\
1,02 \pm 0,02 \\
1,00 \pm 0,02 \\
1,03 \pm 0,02 \\
1,03 \pm 0,02 \\
1,06 \pm 0,03\end{array}$ & $\begin{array}{l}0,91 \pm 0,03 \\
0,96 \pm 0,02 \\
0,97 \pm 0,02 \\
1,02 \pm 0,02 \\
1,03 \pm 0,02 \\
1,06 \pm 0,03\end{array}$ & $\begin{array}{c}1,73 \\
3,46 \\
6,37 \\
9,75 \dagger \\
19,3 \dagger \\
29,0 \dagger\end{array}$ & $\begin{array}{l}6,00 \\
9,70 \\
15,3 \\
20,7 \\
34,2 \\
45,6\end{array}$ & $\begin{array}{l}6,00 \\
9,65 \\
15,1 \\
20,2 \\
32,0 \\
40,7\end{array}$ & $\begin{array}{l}1,04 \pm 0,02 \\
1,03 \pm 0,02 \\
1,03 \pm 0,02 \\
1,06 \pm 0,02 \\
1,10 \pm 0,03 \\
1,08 \pm 0,04\end{array}$ & $\begin{array}{l}0,85 \pm 0,02 \\
0,88 \pm 0,02 \\
0,88 \pm 0,02 \\
0,98 \pm 0,02 \\
1,04 \pm 0,03 \\
1,03 \pm 0,04\end{array}$ \\
\hline
\end{tabular}

Tab. 1. Die experimentellen Halbwertwinkel $\Theta_{\mathrm{H}}$ in Grad und die für die Wegverlängerung korrigierten experimentellen $\Theta_{\mathrm{H}}^{\text {korr }}$ in Grad für $\mathrm{Al}, \mathrm{Ni}, \mathrm{Ag}$, $\mathrm{Au}$ als Funktion von $\varrho x$ in $\mathrm{g} / \mathrm{cm}^{2}$. $\beta$-Energie $680 \mathrm{keV}$. Vergleich

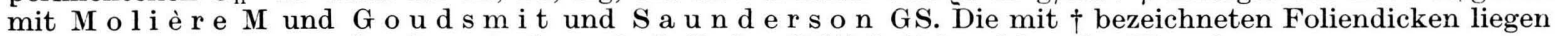
an der Grenze oder außerhalb des Gültigkeitsbereiches der Theorien.

diese Voraussetzungen nicht mehr gelten, weil die Streuwinkel zu groß sind, erhebliche Abweichungen von der Molièreschen Theorie auf (die theoretischen Werte sind deshalb hier nicht eingetragen). Ebenso war die Annäherung durch eine Gauß-Kurve um so schlechter, je stärker die Streuung war. Die in Abb. 3 gestrichelt eingezeichneten Gauß-Kurven sind beim Winkel 0 und beim Halbwertwinkel $\Theta_{\mathrm{H}}$ angepaßt.

Die nach Gl. (4) korrigierten experimentellen Halbwertwinkel $\Theta_{\mathrm{H}}$ sind in Tab. 1, Spalte 2, 7, 12, 17 zusammengestellt. Für den Vergleich dieser Werte

$$
\begin{gathered}
\bar{l}=\int_{0}^{x}(\overline{\mathrm{d} \xi / \cos \vartheta}) \approx \int \mathrm{d} \xi\left(1+\frac{1}{2} \overline{\vartheta^{2}}\right) \\
=\int \mathrm{d} \xi\left[1+\lambda^{2}(\xi)\right] .
\end{gathered}
$$

Nun ist $\lambda^{2}(\xi) \approx k \xi$, wo $k$ eine Konstante ist, also $\bar{l} \approx x\left(1+\frac{1}{2} k x\right)=x\left[1+\frac{1}{2} \lambda^{2}(x)\right] \approx x / \cos \lambda(x)$.

* Diese kurze Herleitung ist nicht ganz einwandfrei, weil die $\vartheta$ nicht statistisch unabhängig sind, doch führt die exakte Rechnung zu demselben Ergebnis. 
Wird also der Halbwertwinkel $\Theta_{\mathrm{H}}$ bei der Schichtdicke $x$ gemessen, so gehört zu der Bahnlänge $x$ etwa der Halbwertwinkel

$$
\Theta_{\mathrm{H}}^{\mathrm{korr}}=\Theta_{\mathrm{H}} \sqrt{x / \bar{l}}=\Theta_{\mathrm{H}} \sqrt{\cos \lambda} .
$$

Diese korrigierten Werte sind in den Spalten 3, 8, 13, 18 der Tab. 1 aufgeführt, ihr Verhältnis zu den theoretischen Werten nach Molière und nach Goudsmit und Saunderson in den Spalten 4, 9, 14, 19 bzw. 5, 10, 15, 20.

Tab. 1 zeigt, daß in den Schichtdickenbereichen, in denen die Theorien Gültigkeit beanspruchen das sind die nicht angekreuzten Messungen - die Molièresche Theorie die Messungen innerhalb der statistischen Genauigkeit durchweg gut wiedergibt. Dasselbe haben Hanson, Lanzl, Lyman und $\mathrm{Scott}^{9}$ für die viel größere Elektronenenergie von 15,7 MeV festgestellt. Die Theorie von Goudsmit und Saunderson dagegen liefert bei hoher Ordnungszahl $(\mathrm{Au}) \mathrm{zu}$ breite Verteilungen, wie auch Kulchitsky und Latyshev ${ }^{8}$ mit Elektronen von 2,25 $\mathrm{MeV}$ gefunden haben. Bei den angekreuzten Messungen, die außerhalb des Gültigkeitsbereiches der Theorien liegen, wird die Übereinstimmung mit Molière offensichtlich durch die Bahnlängenkorrektion

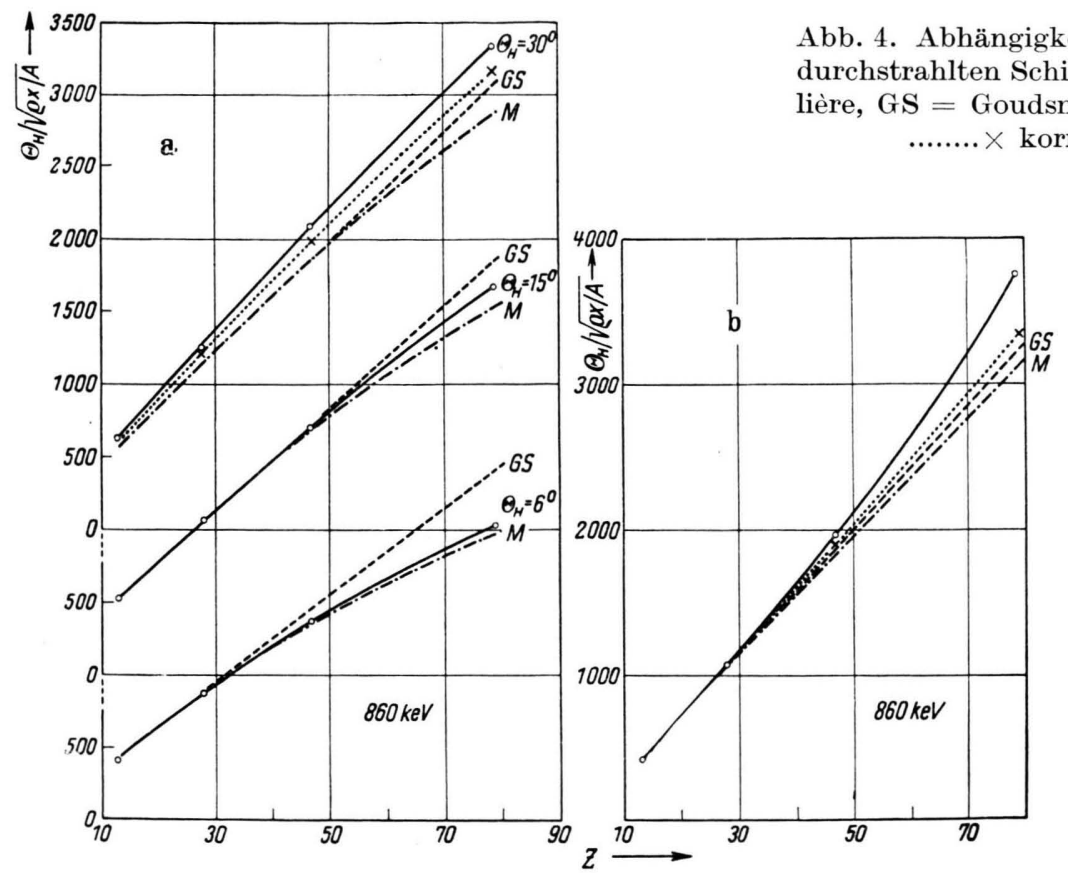

Abb. 5a) $Z$-Abhängigkeit der Vielfachstreuung für streuäquivalente Folien. b) $Z$-Abhängigkeit für Folien gleicher Atomzahl $/ \mathrm{cm}^{2}$. - $\overline{0}$ experimentell, ....... $\times$ exp. korrigiert für Wegverlängerung, .-.. Molière $(\overline{\mathbf{M}}),---$ Goud- $^{-}$ smit $u$. Saunderson (GS). verbessert, ohne jedoch vollkommen $\mathrm{zu}$ werden. Dies wäre auch dann nicht zu erwarten, wenn die Bahnlängenkorrektion genau durchführbar wäre. Hinzu kommt noch die bei den größeren Schichtdicken bereits merklich werdende Geschwindigkeits-
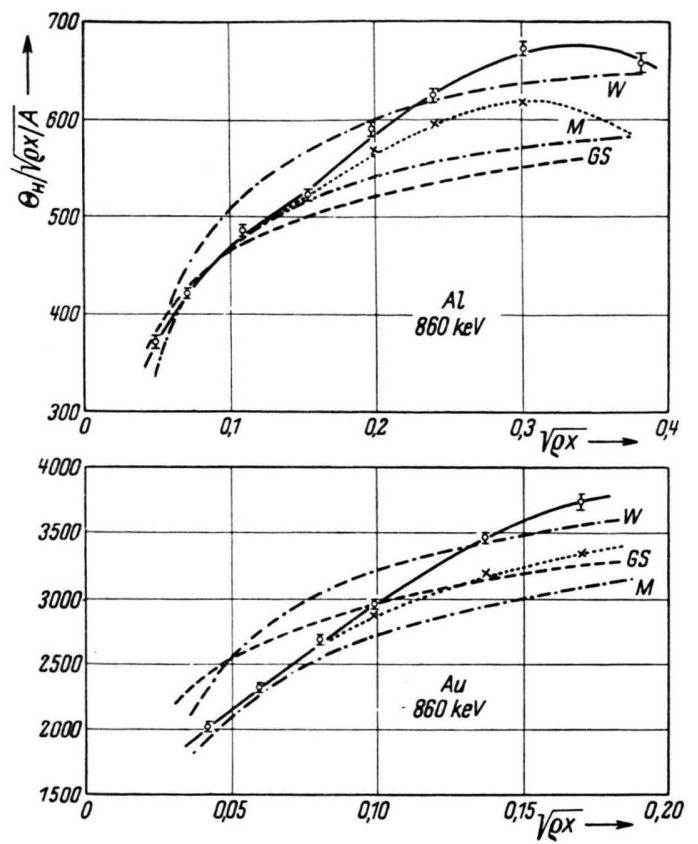

Abb. 4. Abhängigkeit der Vielfachstreuung von der durchstrahlten Schichtdicke. $\mathrm{W}=$ Williams, $\mathrm{M}=$ Molière, GS = Goudsmit u. Saunderson, - $\overline{\bar{O}}$ gemessen, $\times$ korrigiert für Wegverlängerung.

abnahme in der Schicht, die in demselben Sinne wirkt wie die Bahnverlängerung; beide Einflüsse steigern sich sogar gegenseitig.

Charakteristisch ist, daß $\lambda$ und $\Theta_{\mathrm{H}}$ nicht genau proportional $\sqrt{x}$ gehen, wie nach Gl. (2) zu erwarten wäre. Dies wird noch einmal in Abb. 4 gezeigt, wo $\Theta_{\mathrm{H}}(\varrho x / A)^{-1 / 2}$ als Funktion von $(\varrho x)^{1 / 2}$ für $\mathrm{Al}$ und $\mathrm{Au}$ dargestellt ist; nach Gl. (2) wären horizontale Gerade zu erwarten. Das schon über die Theorien von Molière und Goudsmit und Saunderson Gesagte zeigt sich auch hier deutlich. Außerdem sind die theoreti- 
schen Kurven nach Willia $\mathrm{ss}^{2}$ eingetragen, die in ihrem Gültigkeitsbereich deutlich zu hoch liegen, wie auch bei den 2,25 MeV-Messungen von Kulchitsky und Latyshev.

\section{Z-Abhängigkeit der Vielfachstreuung}

Die Abhängigkeit der Vielfachstreuung von der Ordnungszahl $Z$ der Streuschicht kann man nach verschiedenen Gesichtspunkten an Hand der Tab. 1 für $860 \mathrm{keV}$-Elektronen darstellen. Man kann entweder Schichten von gleichem $\Theta_{\mathrm{H}}$ miteinander vergleichen, damit der Wegverlängerungseffekt gleich bleibt; in diesem Falle ist es zweckmäßig, $\Theta_{\mathrm{H}}(\varrho x / A)^{-1 / 2}$ als Funktion von $Z$ aufzutragen, wie in Abb. 5 a geschehen. Oder man vergleicht Schichten mit gleicher Zahl von Atomen je $\mathrm{cm}^{2}$, d. h. gleichem $\varrho x / A$, um etwa die gleiche Zahl von Einzelstreuungen zu haben, wie in Abb. $5 \mathrm{~b}$. Die eingetragenen Meßpunkte sind nach Tab. 1 interpoliert. In beiden Fällen würden sich nach Gl. (2) Gerade durch den Nullpunkt ergeben [abgesehen von einem kleinen Korrektionsfaktor $\left(1+Z^{-1}\right)^{1 / 2}$ bei kleinem $Z$ für die Streuwirkung der Atomelektronen]. In der Krümmung der experimentellen Kurve drückt sich wieder die Inkonstanz von $C$ aus. Von den eingezeichneten theoretischen Kurven in Abb. 5a zeigt die nach Molière die beste Übereinstimmung innerhalb deren Gültigkeitsbereich $\left(\Theta_{\mathrm{H}} \leqq 20^{\circ}\right)$. Bei $\Theta_{\mathrm{H}}=30^{\circ}$ geben beide Theorien zu kleine Werte aus den schon in Ziff. 4 erörterten Gründen.

Abb. 5b ist für $\varrho x / A=1,64 \cdot 10^{-4}$ gezeichnet, was $9,9 \cdot 10^{19}$ Atomen $/ \mathrm{cm}^{2}$ entspricht. Hier besteht wiederum bei genügend kleinen Winkeln, d. h. kleinem $Z$ Übereinstimmung mit Molière und ungefähr ebenso gute mit Goudsmit und Saunderson. Bei großem $Z$ werden aber unvermeidlich die $\Theta_{\mathrm{H}}$ so groß, daß wiederum aus den schon erörterten Gründen keine gute Übereinstimmung mehr erwartet werden darf.

\section{Energieabhängigkeit der Vielfach- streuung}

Die Abhängigkeit des Halbwertwinkels von der Elektronenenergie bei festgehaltener Schichtdicke wurde für eine Al-Folie von $11,6 \mathrm{mg} / \mathrm{cm}^{2}$ und für eine etwa streuäquivalente Au-Folie von $3,46 \mathrm{mg} / \mathrm{cm}^{2}$ in dem Energiebereich 0,25 bis $1 \mathrm{MeV}$ gemessen. Die Ergebnisse für Al sind in Abb. 6 dargestellt. Man erkennt auch hier, daß für $\Theta_{\mathrm{H}} \leqq 20^{\circ}$ die Molièresche Theorie sich bestätigt, während bei kleinen
Energien, wo $\Theta_{\mathrm{H}}$ den Gültigkeitsbereich der Theorie überschreitet, die oben erwähnten Abweichungen auftreten. Nach der einfachen Gl. (2) und nach Goudsmit und Saunderson ergibt sich fast genau dieselbe Energieabhängigkeit wie nach Molière. Die Ergebnisse für die Au-Folie zeigen dasselbe Bild wie die für die Al-Folie in Abb. 6.

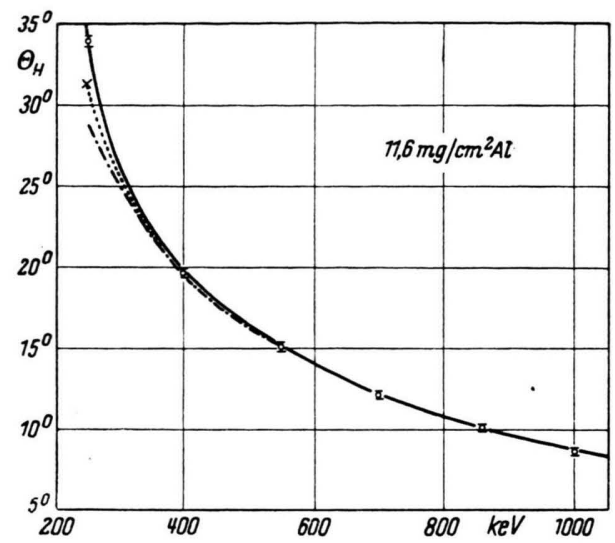

Abb. 6. Der Halbwertwinkel $\Theta_{\mathrm{H}}$ der Winkelverteilungskurven hinter $11,6 \mathrm{mg} / \mathrm{cm}^{2} \mathrm{Al}$ als Funktion der Elektronenenergie. - $\overline{\bar{O}}$ experimentell, ...... korrigiert für Wegverlängerung, -_-_-_ Molière (Absolutwerte).

\section{Kurven für den praktischen Gebrauch}

Die Formeln der neueren Theorien, insbesondere die von Molière ${ }^{4}$ und Snyder und Scott ${ }^{5}$, haben sich zwar als brauchbar erwiesen in beschränkten Bereichen, namentlich bei schwacher Vielfachstreuung, doch sind sie notwendigerweise sehr unhandlich. Praktisch kommt es aber häufig nur darauf an, die wirkliche Verteilung durch eine Gauß-Verteilung nach (1) zu approximieren, was nach unseren Ergebnissen auch bei breiter Winkelverteilung noch recht gut möglich ist, wenn man von dem „Einzelstreuschwanz" absieht. Dann reduziert sich die Frage auf die Ermittlung der häufigsten Ablenkung $\lambda$ oder des Halbwertwinkels $\Theta_{\mathrm{H}}\left(\Theta_{\mathrm{H}}=1,18 \lambda\right)$. Gl. (2) hierfür, mit konstantem Faktor $C$ genommen, hat sich als unzureichend erwiesen; $C$ ist in Wirklichkeit eine langsam veränderliche Funktion der Schichtdicke $x$, der Ordnungszahl $Z$ der Streuschicht und der Elektronenenergie $E$. Man kann aber für viele Fälle ausreichend genaue $\lambda$-Werte nach folgendem Verfahren ermitteln: Man setzt zunächst in (2) für $C$ einen festen Mittelwert, etwa $C=0,65 \mathrm{MeV}$ und berechnet damit $\lambda_{0,65}$. Um das wirkliche $\lambda \mathrm{zu}$ finden, hat man dann noch eine nicht 
sehr große Korrektion anzubringen. Diese Korrektionen sind auf Grund unserer Messungen in Abb. 7 in Prozenten als Funktion von $\lambda_{0,65}$ mit den beiden Parametern Ordnungszahl und Elektronenenergie dargestellt. Die ausgezogenen Kurventeile dürften auf $\leqq 3 \%$ genau sein, die gestrichelten auf $\leqq 6 \%$. Für zwischenliegende $Z$ - und Energiewerte kann man mit dieser Genauigkeit interpolieren. Auch kleinere Extrapolationen für $Z<13$ und $Z>79$ und für Energien $>1 \mathrm{MeV}$ können vorgenommen werden.

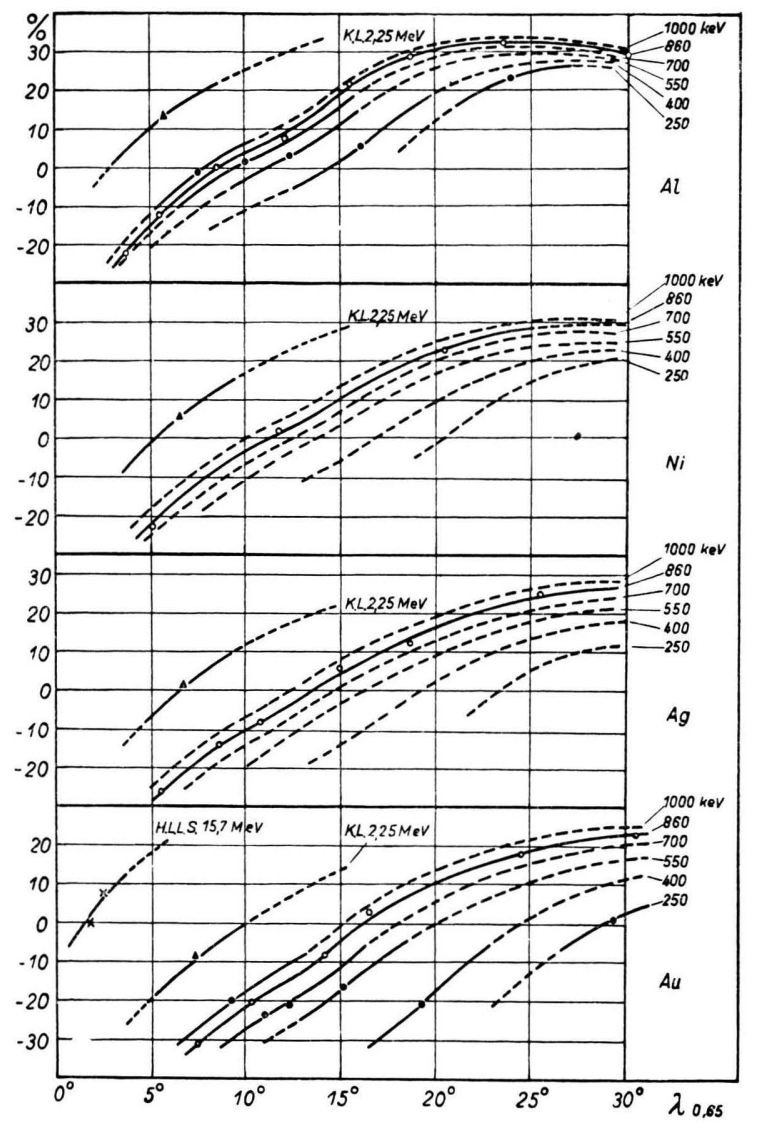

Abb. 7. Korrektionen in $\%$ als Funktion von $\lambda_{0,65}$ zur Ermittlung der wirklichen $\lambda$-Werte $\left(4^{0}<\lambda<35^{\circ}\right)$ für beliebige Folien und Energien $\leqq 1 \mathrm{MeV}$.

Die Kurven der Abb. 7 wurden auf folgende Weise gewonnen: Zunächst wurden in den direkt gemessenen Fällen die Gauß-Kurven ebenso wie in Abb. 3 angepaßt, d. h. bei dem Halbwertwinkel $\Theta_{\mathrm{H}}$, und hieraus $\lambda$ und damit $C$ errechnet. Wegen der vielen Parameter konnten aber solche Messungen nicht in dem ganzen untersuchten $(x, Z, E)$-Bereich gleichmäßig dicht gemacht werden, es mußten Ex- trapolationen vorgenommen werden. Hierbei konnten folgende im Laufe der Untersuchung gefundenen allgemeinen Ergebnisse mit Nutzen verwendet werden (vgl. die $860 \mathrm{keV}$-Kurven für $\mathrm{Al}$ und $\mathrm{Au}$ in Abb. 7):

1. In Übereinstimmung mit allen genaueren Theorien steigt die Korrektion bis zu $\lambda \approx 15^{0}$ verzögert an.

2. Oberhalb von etwa $15^{0}$ wird der Anstieg steiler. Dies ist hauptsächlich auf die Wegverlängerung in der Schicht zurückzuführen (Ziff. 4), daher wird dieses Steilerwerden für alle Materialien etwa bei demselben $\lambda$ beginnen.

3. Bei den größten Winkeln wird der Anstieg wieder flacher oder geht sogar in einen langsamen Abfall über (vgl. Al $860 \mathrm{keV}$ ). Dies hängt offenbar mit der beginnenden Einstellung der ,,vollständigen Diffusion“ zusammen, bei der schließlich $\lambda$ unabhängig von der Schichtdicke wird. Es scheint nach den Messungen, daß sich dieser Umstand bei kleiner Ordnungszahl $Z$ früher bemerkbar macht als bei großem $Z$.

In Abb. 7 sind auch einige andere Messungen eingetragen, die ähnliche Genauigkeit wie die unseren beanspruchen können, nämlich die von Kulchits ky und Latyshev ${ }^{8}$ für $2,25 \mathrm{MeV}$ (K. L.) und die von Hansen, Lanzl, Lyman u. Scott ${ }^{9}$ für 15,7 $\mathrm{MeV}$. (H. L. L. S.). Diese Punkte liegen dort, wo man sie bei vernünftiger Extrapolation nach unseren Kurven erwarten würde und können daher ebenfalls zur Interpolation benutzt werden.

Die Kurven der Abb. 7 können im Prinzip auch für andere einfach geladene Strahlenteilchen der Energie $E$ Verwendung finden. Hierzu berechne man $\lambda_{0,65}$ nach Gl. (2) mit dem entsprechenden $E_{0}$. Sodann berechne man $\lambda_{0,65}^{\mathrm{e}}=\lambda_{0,65} \mathrm{~m} / \mathrm{m}_{\mathrm{e}}(\mathrm{m}=$ Ruhemasse des Teilchens, $m_{\mathrm{e}}=$ Ruhemasse des Elektrons); dies ist die zu wählende Abszisse. Aus der Kurvenschar wählt man die Kurve für die Elektronenenergie $\mathrm{E}^{\mathrm{e}}=E m_{\mathrm{e}} / m$ und findet so die an $\lambda_{0,65}$ anzubringende Korrektion. Diese einfachen Beziehungen folgen aus der allgemeinen Theorie der Vielfachstreuung, indem man Fälle mit gleichem mittleren Stoßparameter für die beiden Teilchen vergleicht. Vorausgesetzt ist hierbei aber, daß $\lambda_{0,65}^{e} \leqq 15^{0}$ (Ziff. 4) bleibt, weil andernfalls durch den Bahnverlängerungseffekt die einfachen Ähnlichkeitsbeziehungen gestört werden. Daher werden praktisch diese Ähnlichkeitsbeziehungen nur in seltenen Fällen von Nutzen sein. 
Wenn die projizierten Streuwinkel gemessen werden (Nebelkammer, photographische Platte), so werden $\lambda$ und $\Theta_{\mathrm{H}}$ um ca. $8-10 \%$ größer; wegen genauerer Formeln vgl. Molière ${ }^{4}$ und $\mathrm{Hanson}$, Lanzl, Lyman und Scott ${ }^{9}$.

\section{Messungen mit schrägen Folien}

Praktisch tritt nicht selten die Frage nach der Winkelverteilung hinter einer Folie auf, die nicht senkrecht zum Primärstrahl steht, sondern deren Normale um einen gewissen Winkel $\alpha$ gegen die Primärrichtung geneigt ist. Für diesen Fall ist bisher nur die Gaußsche Näherung für nicht zu große $\alpha$ berechnet worden ${ }^{10}$ : Gl. (3). Zur Prüfung dieser Theorie wurden die folgenden Messungen mit 700 $\mathrm{keV}$-Elektronen gemacht.

Die Folien wurden in der Folienhalterung FH gedreht (s. Abb. 1). Die Folienhalterung samt der Folie - also die Einfallsebene - konnte um den Primärstrahl gedreht werden (Azimut 0 bei $\varphi=0$; Azimut $90^{\circ}$ bei $\psi=0$ ). Es war somit die ganze räumliche Streuverteilung meßbar. Praktisch wurde das so gemacht, daß für verschiedene Azimute jeweils die ,,Schnittkurven“ der räumlichen Verteilung aufgenommen wurden. Diese Kurven waren im allgemeinen unsymmetrisch, außer natürlich beim Azimut $90^{\circ}$. Ein Beispiel ist in Abb. 8 gezeigt. Die theo-

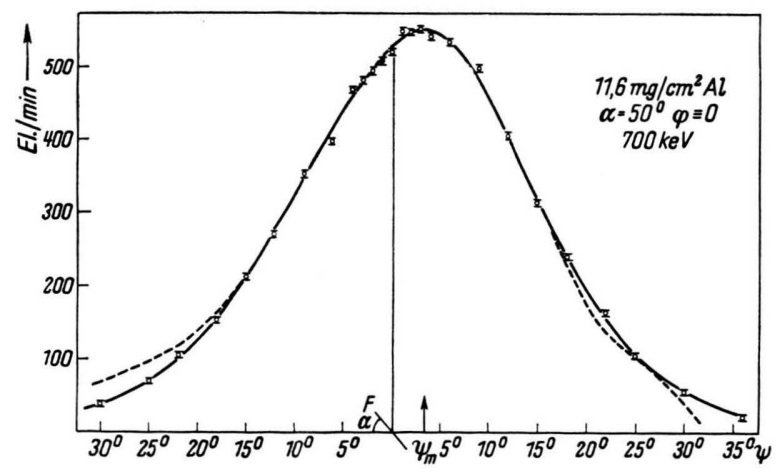

Abb. 8. ,Schnittkurve“" durch die räumliche Streuverteilung in der Einfallsebene hinter einer schrägen

Folie F. - $\overline{\underline{O}}$ experimentell, - - - theoretisch.

retische Kurve nach Gl. (3) ist gestrichelt gezeichnet. Sie weicht nur in den ,Einzelstreuschwänzen“" wesentlich ab, wo die Theorie keinen Anspruch auf Gültigkeit erhebt. Ähnliche Übereinstimmung wurde auch für die anderen Azimute gefunden. Die Verschiebung des Maximums im Sinne der Foliendrehung ist zu erkennen. Nach Gl. (3) sollte näm- lich das Maximum bei $\varphi_{m}=0 ; \psi_{m}=-\lambda^{2} \operatorname{tg} \alpha$ liegen, was sich innerhalb der Meßgenauigkeit bestätigte, sofern $\lambda$ und $\alpha$ in den vorausgesetzten Grenzen blieben; siehe Abb. 10.
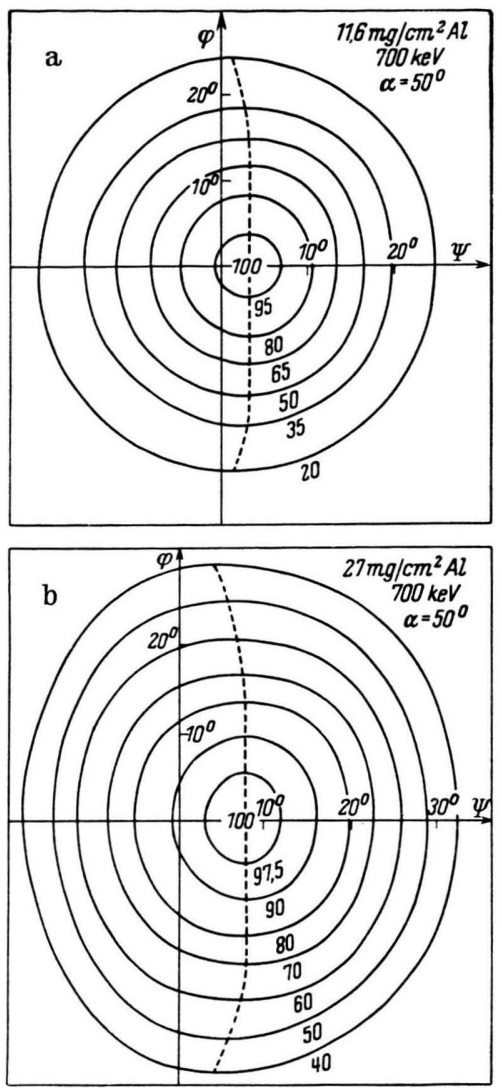

Abb. 9. Räumliche Streuverteilung hinter schrägen Folien, dargestellt durch Höhenlinien.

Die ganze räumliche Verteilung konnte aus den „Schnittkurven" gewonnen werden und durch Höhenlinien (Linien gleicher Intensität) dargestellt werden. Abb. 9 zeigt dies für zwei Fälle. Legt man Schnitte durch die glockenförmige Verteilung parallel zur $\psi$-Achse, so sollten die Maxima dieser „,Schnittkurven“ auf den gestrichelt gezeichneten Kurvenstücken liegen. Dies ist innerhalb der Fehlergrenzen der Fall.

\section{Diskussion}

Die Theorie der Vielfachstreuung von Molière ${ }^{4}$ und die zu den gleichen Ergebnissen führende Theorie von Snyder und Scott ${ }^{5,6}$ werden durch die vorliegenden Messungen im Bereich mäßiger Streu- 
breiten durchweg bestätigt. Die Voraussetzungen dieser Theorien erweisen sich damit als gerechtfertigt; es sind dies: vernachlässigbare Umwege in der Folie, verschwindend kleiner Energieverlust in der Streufolie, verschwindende Einflüsse des Elektronenspins, der Gitterstruktur feinkristalliner Metallfolien und der endlichen Ausdehnung der Atomkerne bei den hier verwendeten Elektronenenergien.
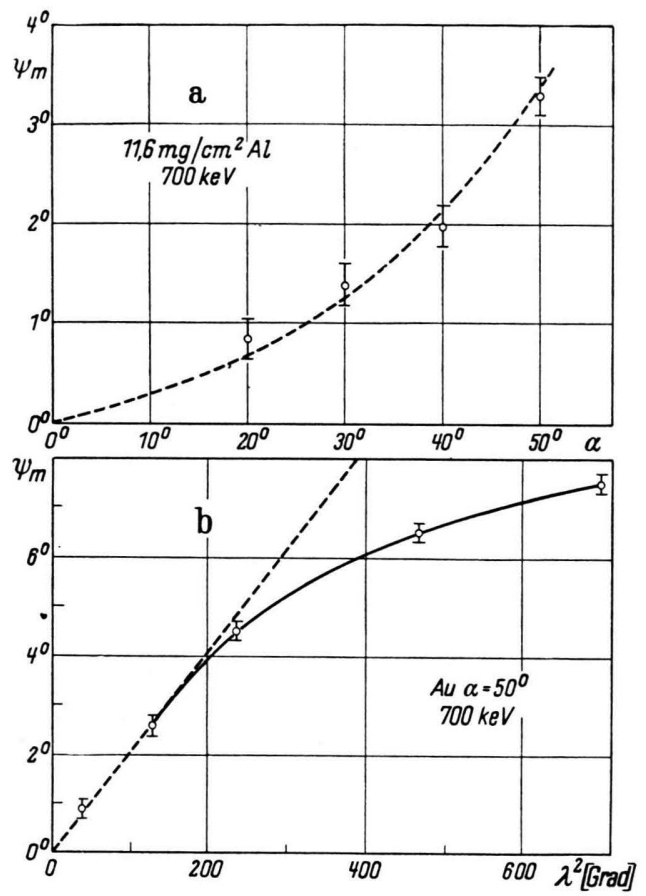

Abb. 10 a. Maximumverschiebung $\psi_{m}$ als Funktion des Winkels $\alpha$ (Schrägstellung).

Abb. $10 \mathrm{~b}$. Maximumverschiebung $\psi_{m}$ bei veränderlicher Schichtdicke als Funktion von $\lambda^{2} . \lambda$ in Grad. $\overline{\text { ㅇ }}$ experimentell, - - - theoretisch.

Die Theorien von Williams ${ }^{2}$ und Goudsmit und Sa underson ${ }^{3}$ liefern bei schweren Elementen deutlich zu große Streubreiten. Dies dürfte im wesentlichen daher rühren, daß hier die Abschirmung des Atomkernes durch die Hüllenelektronen noch nicht so vollkommen berücksichtigt ist wie bei Molière.

Bei dickeren Folien, d. h. bei Halbwertwinkeln $>$ etwa $20^{\circ}$, wird keine der bestehenden Theorien den Messungen gerecht, weil die theoretischen Voraussetzungen z.Tl. nicht mehr erfüllt sind. Insbesondere werden die Umwege der Elektronen in der Folie beträchtlich, daher wird die Bahnlänge wesentlich größer als die Foliendicke. Dieser Einfluß läßt sich nach Ziff. 4 abschätzen. Gleichzeitig setzt auch Rückdiffusion merklich ein. Schließlich sind auch die Energieverluste in der Folie nicht mehr zu vernachlässigen. Alle diese Faktoren wirken im Sinne einer Verbreiterung der Streuverteilung und sind so miteinander gekoppelt, daß sie sich gegenseitig verstärken. Bei sehr großen Schichtdicken stellt sich dann die ,,vollständige Diffusion“ mit konstanter Streubreite ein, die erst recht nicht von den erwähnten Theorien erfaßt wird. Eine erweiterte Theorie, die alle diese Faktoren berücksichtigt, wäre notwendig sehr verwickelt und liegt bisher nicht vor. Aus diesem Grunde wurden in Abb. 7 einige empirische Unterlagen gegeben, um in diesem Bereich wenigstens die Halbwertwinkel mit einer für die meisten $Z$ wecke ausreichenden Genauigkeit zu ermitteln.

Liegt die Streufolie nicht senkrecht, sondern schräg zum einfallenden Strahl, so werden naturgemäß die Verhältnisse noch verwickelter, weil die Axialsymmetrie des gestreuten Bündels entfällt. Theoretisch liegt hier nur die Gaußsche Näherung ${ }^{10}$ vor, die innerhalb ihrer Gültigkeitsgrenzen durch die Messungen bestätigt wurde, insbesondere hinsichtlich der Verschiebung des Intensitätsmaximums.

Der Deutschen Forschungsgemeinschaft danken wir für die Bereitstellung von Apparaten. 